
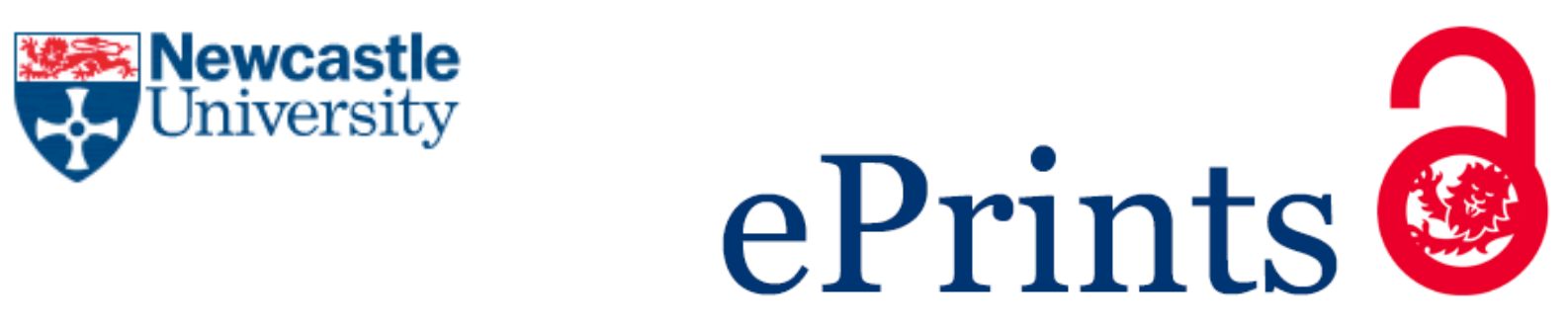

Pippard BJ, Shipley MD.

Healthcare staff attitudes towards the use of electronic cigarettes

('e-cigarettes') compared with a local trust policy.

Perspectives in Public Health (2016)

DOI: $\underline{\text { http://dx.doi.org/10.1177/1757913916659311 }}$

\title{
Copyright:
}

This is the authors' accepted manuscript of an article that has been published in its final definitive form by Sage Publications Ltd, 2016.

DOI link to article:

http://dx.doi.org/10.1177/1757913916659311

Date deposited:

$26 / 10 / 2016$

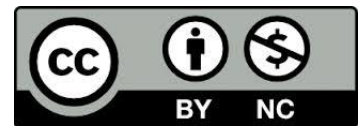

This work is licensed under a Creative Commons Attribution-NonCommercial 3.0 Unported License 
Healthcare staff attitudes towards the use of electronic cigarettes ('e-cigarettes') compared with a local trust policy

B J Pippard (BSc., MRes., MBBS, MRCP)

Clinical Teaching Fellow

Newcastle upon Tyne Hospitals NHS Foundation Trust

and

M D Shipley (MBBS, MRCP)

Consultant Respiratory Physician

South Tyneside NHS Foundation Trust 


\section{Healthcare staff attitudes towards the use of electronic cigarettes ('e-cigarettes') compared with a local trust policy}

\section{Introduction}

The use of electronic cigarettes ('e-cigarettes') has risen dramatically in recent years. In 2015, an estimated 2.6 million adults in the UK were regularly using these devices, ${ }^{1}$ and they are now reportedly the most widely used product for smoking cessation. ${ }^{2}$ Despite this growth in popularity, considerable debate remains over the acceptability of ecigarettes, not least in relation to concerns about unknown long-term health risks. Opponents (including a number of professional bodies) are cautious about encouraging widespread use, given the paucity of current evidence. The potential for 'normalising' smoking behaviour, particularly among young people, has also been widely discussed. ${ }^{3,4}$ However, the possible harm reduction compared to smoking tobacco products is a major driving force for those pursuing their use as a viable public health initiative. A recent report ${ }^{2}$ by Public Health England concluded that e-cigarettes were $95 \%$ safer than standard cigarettes, based on the relative amounts of constituent chemicals in these products. Additionally, a Cochrane review suggests e-cigarettes may be effective aids to smoking cessation, ${ }^{5}$ although they remain, at present, largely unregulated.

Currently in the UK, it is not illegal to use e-cigarettes within enclosed public places. Nonetheless, a number of organisations - including the vast majority of NHS 
Trusts - have adopted restrictions on use consistent with the 2007 ban on smoking tobacco products. Specifically, at South Tyneside NHS Foundation Trust, existing 'Smokefree' regulations have been extended to include e-cigarettes, such that staff, patients and visitors are not permitted to use these devices anywhere on Trust premises, with the exception of designated smoking shelters. ${ }^{6}$ It is the responsibility of all healthcare staff working in the Trust to help enforce this policy, although the basis for imposing such restricted use is not clear. Moreover, the successful implementation of this policy remains uncertain.

The present study examined staff attitudes towards the use of e-cigarettes in a hospital environment (South Tyneside District Hospital, STDH) with respect to enforcement of a local Trust smoking policy. In particular, factors influencing the likelihood of individuals challenging use of e-cigarettes on Trust premises were assessed.

\section{Methods}

A questionnaire was delivered opportunistically to full-time members of staff working on medical and surgical wards at STDH over a period of five days during May 2015. Verbal consent was obtained following a brief explanation of the purpose of the study. Participants were asked to complete the questionnaire and return it in a sealed envelope provided, to maintain confidentiality. Respondents were asked to indicate their personal use of e-cigarettes (ever user, never user, or regular user) and whether they thought they 
should be permitted in public places. In addition, they were asked to indicate whether they thought patients, visitors, or members of staff should be allowed to use e-cigarettes on hospital grounds, or within hospital buildings (e.g. wards), respectively. Respondents were asked if they were aware of a Trust policy regarding e-cigarettes (no; yes, and had read it; yes, but had not read it), whether they had ever challenged a patient, visitor or member of staff using an e-cigarette and what, if anything, would prevent them from doing so. Space was provided within the questionnaire for free-text comments, including perceived risks of using e-cigarettes within a hospital environment.

Qualitative and quantitative analysis of data was performed by BP. The chisquared test was used to determine whether significant differences ( $p$-value <0.05) existed between subgroups of respondents.

\section{Results}

\section{Demographics:}

79/85 questionnaires were completed (response rate 93\%), comprising fifty-eight (73\%) females and twenty-one (27\%) males (age range 19-59; mean 33.8 years). Thirty-six $(46 \%)$ were medical staff, thirty (38\%) nursing staff, and thirteen (16\%) allied healthcare professionals. 12/78 (15.5\%) were current smokers, twelve (15.5\%) ex-smokers, and fifty-four (69\%) never smokers (one person did not disclose their smoking status). 12/79 (15\%) had previously tried an e-cigarette, three of whom were self-reported 'never 
smokers'. None of the respondents were regular e-cigarette users. The majority (67/79, $85 \%)$ had never used an e-cigarette.

Views regarding use of e-cigarettes:

$34 / 75$ respondents $(45 \%)$ thought e-cigarettes should be allowed in public places (four people chose not to answer this question). 65/79 respondents (82\%) thought no-one should be allowed to use e-cigarettes within hospital premises (e.g. wards); thirty respondents $(38 \%)$ thought no-one should be allowed to use e-cigarettes on hospital grounds (Figure 1). There were no significant differences in response according to smoking status, professional role, sex, age, or awareness of policy.

\section{[Insert Figure 1.]}

Awareness of policy and challenging use:

59/79 respondents $(75 \%)$ had never challenged someone using an e-cigarette (Figure 1). 'Non-medical' staff (nursing staff and allied healthcare professionals together) were more likely to have challenged patients using an e-cigarette than 'medical' staff ( $\mathrm{p}=0.027$; $12 / 43$ v. $3 / 36$, respectively); there were no other significant differences in challenging patients, visitors, or members of staff according to respondents' smoking status, professional role, sex or age. 43/76 respondents (57\%) were unaware of trust policy on 
the use of e-cigarettes; of those that were aware, only 16 had read it (21\%). Respondents who were aware of the policy were more likely to have challenged patients compared with those who were unaware $(\mathrm{p}=0.001 ; 12 / 33$ v. $3 / 43)$; there was no significant difference with respect to challenging visitors or members of staff.

Perceived risks of e-cigarette use and factors influencing challenge of use:

The main perceived risks of use included: fire hazard (29\%); unknown health risks (27\%); 'normalising' smoking behaviour (20\%); and 'bad image' (18\%). Fear of abuse (25/55, $45 \%)$ and uncertainty about policy/legislation $(11 / 55,20 \%)$ were the dominant reasons preventing people challenging e-cigarette use; only 4/55 (7\%) stated they had never encountered someone using a device.

\section{Discussion}

Most staff did not enforce trust policy on the use of e-cigarettes, with only $25 \%$ respondents having ever challenged someone using a device. This is unlikely to represent a lack of opportunity, as only a small proportion of respondents stated they had never encountered someone using a device within the Trust. Notably, over half respondents were unaware that the Trust even had a policy regarding e-cigarettes, and were less likely to have challenged someone than those who were aware. Despite this, several respondents who were unfamiliar with the policy reported they had previously challenged someone, 
suggesting personal beliefs about (non-) acceptable use may be an important factor in deciding whether to challenge someone or not. Being made aware of the policy may have influenced intention to challenge e-cigarette use in future (Figure 1), highlighting the potential benefit of educating staff to aid policy implementation. Whether reported planned behaviour will translate to actual practice is, however, uncertain. Roughly 1/3 respondents stated they would still not challenge someone using an e-cigarette despite being informed of Trust policy, indicating awareness is only part of the issue.

Fear of abuse was the most cited reason for not challenging e-cigarette use, in agreement with previous findings evaluating the enforcement of NHS Smokefree regulations. ${ }^{7}$ Avoiding potential conflict may, therefore, have a greater influence on successful implementation of policy than whether or not people are aware of - or, even agree with - it. This has particular implications for challenging other staff members, where maintaining good professional relationships may be an overriding factor: it is perhaps more socially acceptable to promote a 'smokefree' image to patients receiving medical care than members of staff or visitors, both of whom were challenged less frequently. It is not clear why 'non-medical' staff should have challenged patients more than 'medical' staff, though the relatively small number of respondents involved may impact these findings.

Respondents were generally more open to e-cigarette use on hospital grounds than within hospital buildings (with only 2 people specifying use within designated shelters), 
despite 55\% stating e-cigarettes should not be allowed in public places. This may reflect a tendency of healthcare staff to accept attempts at smoking cessation by individuals who would otherwise be forced to use devices in shelters alongside other smokers. In contrast, the risks of using e-cigarettes may be more apparent within hospital buildings; specifically, the recognised fire hazard associated with charging devices ${ }^{8}$ was identified as a dominant risk by respondents. Other expressed concerns included uncertain health risks, promoting a 'bad image' and 'normalising' smoking behavior, all issues that are likely to influence current decisions to include e-cigarettes in existing 'Smokefree' policies - even in the absence of smoke - rather than promoting use as a potential cessation device.

\section{Conclusion}

Most staff do not enforce Trust policy regarding e-cigarette use. This reflects variation in opinion over use, poor awareness of the policy itself, and perceived barriers to implementation, especially fear of abuse. Addressing these issues through staff education sessions, including managing conflict, may be beneficial. Future policy development regarding e-cigarette use should be clearly based on the available evidence, taking account of the practicalities of any implementation. 


\section{Limitations}

This study is limited by the particular demographic of respondents working at STDH, the majority of whom were never smokers who had not previously used an e-cigarette. The relatively small sample size limits generalisation of any differences that might exist between the various subgroups. 


\section{References}

1. Action on Smoking and Health. Use of electronic cigarettes in Great Britain. http://ash.org.uk/files/documents/ASH_891.pdf (2014, accessed 10 November 2015)

2. McNeil A, Brose LS, Calder R et al. E-cigarettes: an evidence update. A report commissioned by Public Health England.

www.gov.uk/government/publications/e-cigarettes-an-evidence-update $(2015$, accessed 13 November 2015)

3. Hughes K, Bellis M, Hardcastle K, et al. Associations between e-cigarette access and smoking and drinking behaviours in teenagers. BMC Public Health 2015; 15:244. Doi: 10.1186/s12889-015-1618-4

4. Primack BA, Soneji S, Stoolmiller M, et al. Progression to traditional cigarette smoking after electronic cigarette use among US adolescents and young adults. JAMA Pediatr 2015; 169 (11):1018-1023.Doi:10.1001/jamapediatrics.2015.1742 
5. McRobbie H, Bullen C, Hartmann-Boyce J, et al. Electronic cigarettes for smoking cessation and reduction. Cochrane Database Syst Rev 2014;

12:CD010216. Doi: 10.1002/14651858.CD010216.pub2

6. South Tyneside NHS Foundation Trust. Smoking Policy. PP18 (version 5), March 2015.

7. Shipley M and Allcock R. Achieving a smoke-free hospital: reported enforcement of smoke-free regulations by NHS health care staff. J Public Health $2008 ; 30(1): 2-7$

8. Department of Health Estates and Facilities Alert. E-cigarettes, batteries and chargers. EFA/2014/002, 16 June 2014. 


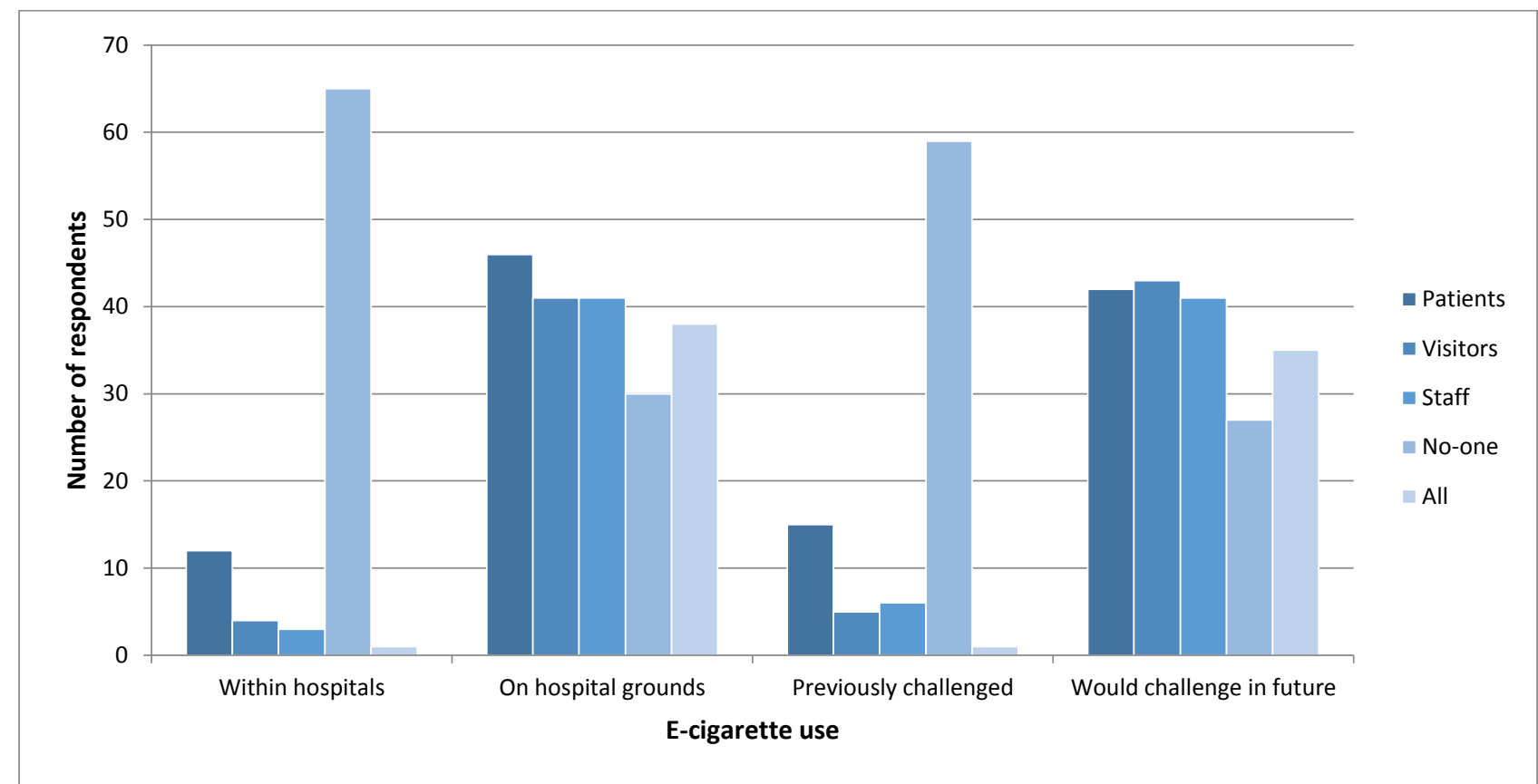

Figure 1: Staff responses regarding acceptable use of e-cigarettes within hospitals or on hospital grounds, and on challenging use, according to user subgroup 